\title{
Relevancia del estatus menopáusico en las diferencias en salud mental entre mujeres y hombres
}

\author{
Maria-Pilar Matud*, Maria-Concepcion Correa*, Juan-Manuel Bethencourt* y Maria-Jose del Pino**
}

* Universidad de La Laguna. Tenerife (España)

**Universidad Pablo de Olavide. Sevilla (España)

\begin{abstract}
Resumen: Se analizan las diferencias entre mujeres y hombres en autoestima y en sintomatología depresiva, de ansiedad y somática, estudiando la relevancia del estatus menopáusico y la edad en tales diferencias. Se utilizó un diseño transversal con una muestra de 1341 personas adultas $(726 \mathrm{mu}$ jeres y 615 hombres) de la población general con diferentes edades, y estatus menopáusico en el caso de las mujeres, quienes respondieron al Cuestionario de Salud General de Goldberg y al Cuestionario de Autoestima de Rector y Roger. Se encontró que las mujeres tenían más síntomas somáticos y de ansiedad que los hombres. No se encontraron diferencias en función del género en depresión ni en autoestima entre los más jóvenes, pero a partir de los 40 años las mujeres tenían más síntomas depresivos y menor autoestima que los hombres con edades similares. Los análisis intragénero evidenciaron que los hombres más jóvenes tenían más sintomatología depresiva y menos autoestima que los de más de 40 años, diferencias intragrupo que no se observaron entre las mujeres. Solo se encontraron diferencias entre las mujeres en función del periodo menopáusico en sintomatología somática, que era mayor en las perimenopáusicas respecto a las premeno-páusicas. Se concluye que la menopausia tiene escasa relevancia en las diferencias de género en salud mental.

Palabras clave: género; menopausia; ansiedad; depresión; sintomatología somática; autoestima.
\end{abstract}

\section{Introducción}

Son muchas las investigaciones que constatan la existencia de diferencias en salud mental entre mujeres y hombres si bien las razones de ello y las condiciones que hacen que tales diferencias se atenúen o aumenten aún están sujetas a debate (cfrs. Emslie et al., 2002; Seedat et al., 2009; Weich, Slogget y Lewis, 1998). En estudios epidemiológicos realizados en diversos países se ha encontrado de forma consistente diferencias en función del género en trastornos mentales, siendo más comunes en las mujeres los afectivos y de ansiedad y en los hombres los de personalidad antisocial y consumo de sustancias (Dawson, Goldstein, Moss, Li y Grant, 2010; Eaton et al., 2012; Kessler et al., 1994; Regier et al., 1988; Seedat et al., 2009). Los trastornos de ansiedad y del humor son los más comunes en la población y frecuentemente coocurren (Kessler, Chiu, Demler y Walters, 2005) lo que puede indicar un mayor riesgo para la salud mental de las mujeres. Se ha encontrado que es más común en las mujeres que en los hombres la morbilidad psiquiátrica menor (Emslie et al., 2002), siendo también más común que informen de sintomatología somática, efecto que parece ser independiente de la comorbilidad psiquiátrica (Kroenke y Spitzer, 1998).

* Dirección para correspondencia [Correspondence address]: M. Pilar Matud. Facultad de Psicología. Universidad de La Laguna. Campus de Guajara, 38205 La Laguna, Tenerife (España).

E-mail:pmatud@ull.es
Title: Relevance of menopausal status in mental health differences between women and men.

Abstract: The objective of this study is to examine differences between men and women in self-esteem and depressive, somatic and anxiety symptoms when studying the relevance of menopausal status and age in such differences. Research design was a cross-sectional survey of a general population sample of 1341 ( $n=726$ women and $n=615$ men) adults of different ages and, in the case of women, with different menopausal status. Participants responded to Goldberg General Health Questionnaire and Rector and Roger Self-esteem questionnaire. Results showed that women had more anxiety and somatic symptoms than men. Gender differences in depressive symptoms and self-esteem were not found in younger participants, although women over 40 showed more depressive symptoms and lower self-esteem than men of similar ages. Intra-gender analysis indicated that younger men had more depressive symptoms and less self-esteem than those over 40, whereas these differences were not observed in the women sample. Differences among women were only observed in relation with their diverse menopausal status in somatic symptoms, these latter being greater in perimenopausal than in premenopausal women. These results make clear that menopause has little relevance in gender differences in mental health.

Key words: gender; menopause; anxiety; depression; somatic symptoms; self-esteem.

Las hipótesis planteadas para explicar las diferencias de género en salud mental han sido diversas, habiéndose propuesto como agentes causales desde factores psicosociales a biológicos. Dentro de estos últimos destacan los que han tratado de atribuir las diferencias entre mujeres y hombres en salud mental al efecto de las hormonas sexuales. Tal y como plantea Ussher (1992), durante siglos se ha considerado el ciclo reproductivo femenino como un determinante principal de la conducta de las mujeres y, tanto en el discurso popular como en el académico, se ha planteado la existencia de síndromes como el premenstrual, el postnatal y el menopáusico a los que se ha considerado como las causas subyacentes de la ansiedad y depresión en las mujeres (cfrs. Payne, Palmer y Joffe, 2009), así como de una serie de síntomas físicos y de otras diferencias en salud y conducta entre mujeres y hombres. Desde esta perspectiva se ha hipotetizado la existencia de una susceptibilidad biológica planteándose que la variación cíclica neuroendocrina implicada en la reproducción femenina es vulnerable al cambio. Y se afirma que la aparición repentina de altos niveles de estrógenos durante la pubertad altera la sensibilidad de los sistemas de neurotransmisores y el flujo constante, durante los años reproductivos, en los niveles de estrógenos y progesterona augura una modificación constante en los sistemas de neurotransmisores (Steiner, Dunn y Born, 2003). Pero, pese a tales predicciones, aún no se ha encontrado una relación directa entre síntomas psiquiátricos y cambios hormonales tales 
como el descenso de estrógenos (Llaneza, García-Portilla, Llaneza-Suárez y Armott, 2012).

El modelo biomédico ha predominado durante el siglo XX obviándose las múltiples diferencias que existen entre mujeres y hombres en socialización y en roles. Ello parece ser especialmente preminente en la menopausia donde el "modelo biomédico de la menopausia" se ha equiparado con la "biología de la menopausia" (Derry, 2002, p. 13), enfatizando la deficiencia de estrógenos y sobreestimando la centralidad de la enfermedad en la comprensión de la menopausia, la cual se asocia con enfermedad crónica y con pérdida de valor personal, considerando que se da una devaluación física, psicológica y social (Lachowsky, 2002) y se plantea que la profesión médica tiene un papel importante en la restauración de la confianza de las mujeres en sí mismas "con palabras así como con medicamentos" (p. 25).

Pese a que existe una cierta confusión terminológica, generalmente la menopausia alude a la fase de la vida en que cesa el potencial reproductor de la mujer por la finalización de la función ovárica y el cese permanente de la menstruación (Sherman, 2005; Utian, 2004). La Organización Mundial de la Salud (OMS) propone que el término menopausia se defina "como el cese permanente de las menstruaciones que resulta de la pérdida de la actividad folicular ovárica" (1981, p. 10). En torno a dicho periodo se ha utilizado una serie de términos que han pretendido organizar en estadios el proceso de envejecimiento reproductivo de las mujeres y sobre el que ha existido cierto desacuerdo. La OMS convocó en 1980 a un grupo de científicos que hizo una serie de recomendaciones terminológicas, las cuales fueron revisadas y publicadas posteriormente (OMS, 1996) y son las que se utilizan en el presente trabajo.

Según la OMS (1996) el término perimenopausia incluye el periodo inmediatamente anterior a la menopausia, es decir, desde el comienzo de las manifestaciones endocrinológicas, biológicas y clínicas que la preceden, hasta el primer año siguiente a la menopausia. Proponen que el término transición menopáusica se reserve para el periodo que precede al período menstrual final y recomiendan que se use el término postmenopausia para el periodo que comienza a partir de la menopausia, si bien dicho periodo solo puede determinarse cuando han pasado 12 meses consecutivos de amenorrea. Además, reconocieron la ambigüedad en el uso del término premenopausia, el cual se refiere al año o dos años anteriores a la menopausia o bien para la totalidad del periodo reproductivo previo a ésta, recomendando que se utilice para referirse a todo el periodo fecundo hasta el último periodo menstrual.

Se ha planteado que, como consecuencia de los cambios derivados del envejecimiento del ovario y de la función del eje ovario-hipófisis-hipotálamo, la transición menopáusica conlleva un periodo de cambios en los tejidos reproductivos y no reproductivos (Sherman, 2005) a los que se ha atribuido un papel central en la etiología de una serie de síntomas tales como sofocos, sudores nocturnos, atrofia vaginal, cambios de humor, trastornos del sueño y disfunción sexual, si bien actualmente existe una importante controversia respecto a si tales síntomas pueden ser atribuidos de forma fiable a la menopausia (Judd, Hickey y Bryant, 2012; Pimenta, Leal, Maroco y Ramos, 2012). Se ha encontrado que las características sociodemográficas, el estilo de vida y los problemas de salud concomitantes parecen ser determinantes importantes de los síntomas relacionados con la menopausia (Bromberger y Kravitz, 2011; Li et al., 2003; Ishizuka, Kudo y Tango, 2008) y que pueden ser correlatos más importantes de la sintomatología depresiva que se da en la mediana edad que el estatus menopáusico y los niveles hormonales (Gallicchio, Schilling, Miller, Zacur y Flaws, 2007). Además, en la sintomatología asociada a la menopausia también parecen ser importantes las actitudes (Ayers, Forshaw y Hunter, 2010) y las percepciones socioculturales, que constituyen el contexto en el que las mujeres experimentan la menopausia (Hall, Callister, Berry y Matsumura, 2007).

$\mathrm{La}$ edad en que tiene lugar la menopausia en la mayor parte de las mujeres oscila entre los 44 y 55 años, situándose la mediana entre los 50 y 52 (Lund, 2008), si bien se dan algunas variaciones en función de factores culturales y geográficos así como de otros de tipo obstétrico y de conductas tales como el fumar (OMS, 1996). En España, la edad media de la menopausia natural es de 51.4 años comenzando la perimenopausia entre los 47 o 48 años y durando entre dos y cinco años (Mendoza et al., 2013). Tal y como se afirma en el informe del grupo de científicos de la OMS de las investigaciones sobre la menopausia en los años noventa (OMS, 1996), en la menopausia los ovarios dejan de funcionar y disminuye su producción de esteroides y péptidos hormonales. Ello genera en el organismo una serie de cambios fisiológicos, algunos de los cuales son el resultado del cese de la función ovárica y de los fenómenos relacionados mientras que otros son debidos al proceso de envejecimiento. El proceso de envejecimiento, además de implicar cambios biológicos, se acompaña de una serie de cambios complejos asociados con la edad respecto a la percepción que las personas tienen de sí mismas, el valor asignado por las demás y los roles sociales desempeñados (Gázquez, Yuste, y Pérez, 2005). Pero ello no parece reconocerse a nivel popular, donde existe el estereotipo de la mujer menopáusica, cuyo contenido es negativo (Castaño y Martínez-Benlloch, 1990; MarcusNewhall, Thompson y Thomas, 2001). Y tal problema parece ser importante en España donde la menopausia se asocia en muchas mujeres con una variedad de síntomas y preocupaciones sobre su salud futura (Larroy y Gutiérrez, 2009; Mendoza et al., 2013) y donde se detecta que las mujeres de mediana edad tienen "una posición claramente más desfavorable en términos de salud y calidad de vida" (García-Vega, 2011, p. 286).

Por tanto, el objetivo del presente trabajo es conocer si existen diferencias entre mujeres y hombres en la sintomatología mental más común en la población y en autoestima, analizando la relevancia que tienen el estatus menopáusico y la edad en tales diferencias. En la medida en que la menopausia se asocia con la edad y con el proceso de envejeci- 
miento, para conocer la relevancia que la menopausia tiene en las diferencias entre mujeres y hombres en salud mental parece importante analizar, además de la sintomatología mental de las mujeres en las diferentes etapas relacionadas con la menopausia, la de las mujeres más alejadas en edad de dicho periodo, así como la de los hombres con edades similares a las de las mujeres en cada subgrupo estudiado.

\section{Método}

\section{Participantes}

La muestra está formada por un total de 1341 personas, de las que el $45.9 \%(n=615)$ son hombres y el $54.1 \%(n=$ 726) mujeres. Aunque sus edades están comprendidas entre los 20 y 65 años, no se incluyeron personas cuyas edades estuviesen entre 32 y 40 años con la finalidad de lograr mayor diferenciación entre las personas más jóvenes y el resto de los grupos. La media de edad de los hombres es de 44.18 $(D T=11.61)$ y la de las mujeres $44.09(D T=10.63)$, diferencias que no son estadísticamente significativas, $t(1339)=$ $0.14, p=.89$. Su estado civil es diverso, aunque la mayor parte (el 74.6\%) están casadas. El 13.4\% están solteras, casi la décima parte (el 9.1\%) divorciadas o separadas y es mucho menos frecuente que hayan enviudado, lo que solo sucede en el $2.9 \%$. Su número de hijos/as oscila entre 0 y 8 , siendo la media de 1.93 , la desviación típica de 1.42 y la mediana y la moda de 2. Casi la mitad únicamente ha cursado estudios básicos (el 42.3\%), el 28.3\% tiene estudios medios y el 29\% universitarios. También su profesión es diversa, siendo en casi la tercera parte (el 31.1\%) de tipo manual, en el $23.4 \%$ es de tipo no manual y el $25.4 \%$ son profesionales para cuyo ejercicio se precisa titulación universitaria. El 20.1\% ( $n=$ 267) de las mujeres no trabajaba fuera del hogar y se autodefine como "ama de casa".

\section{Instrumentos}

La evaluación de la sintomatología mental se llevó a cabo mediante la versión en Escalas del Cuestionario de Salud General de Goldberg (GHQ-28; Goldberg y Hillier, 1979). Los ítems que lo componen se refieren fundamentalmente a trastornos de tipo afectivo-emocional y consta de 4 subescalas, formadas cada una por 7 ítems, denominadas síntomas somáticos, ansiedad e insomnio, disfunción social, y depresión grave, que representan dimensiones de sintomatología y no son totalmente independientes entre sí (Goldberg, Williams, Lobo y Muñoz, 1996). La versión utilizada ha sido la validada y traducida al español por estos autores y se ha utilizado la puntuación tipo Likert, que asigna pesos desde 0 (para la ausencia de sintomatología) hasta 3 (para la mayor gravedad de cada síntoma). El rango de puntuación en cada una de las cuatro escalas oscila entre 0 y 21 indicando mayor puntuación más sintomatología de cada tipo (somática, depresiva y de ansiedad). En el caso de la escala de disfunción social mayor puntuación indica menor actividad, más insatis- facción y menor capacidad de tomar decisiones. En la muestra del presente trabajo la consistencia interna (alpha de Cronbach) de la escala de síntomas somáticos fue de .83 , la de ansiedad e insomnio .89, la de depresión grave .88 y la de disfunción social .72.

La autoestima se evaluó mediante la versión española del Self-Esteem Inventory, instrumento diseñado y validado por Roger y su equipo en la Universidad de York (Rector y Roger, 1993). Está formado por 43 ítems con un formato de respuesta de cuatro alternativas (desde "nunca", puntuado como 0 , hasta "siempre" que se puntúa con 3) que reflejan la valoración de varias competencias, incluidas la personal, interpersonal, familiar, de logro, atractivo físico, y valoración del grado de incertidumbre de las mismas. En la validación realizada en Canarias con una muestra de 3728 personas de la población general (Matud, Ibáñez, Marrero y Carballeira, 2003) se encontraron dos factores cuya estructura factorial era independiente del género: valoración negativa de sí mismo/a, formado por 28 ítems con una consistencia interna de .91; autoconfianza, formado por 15 ítems que recogen afirmaciones que reflejan seguridad y confianza en sí mismo/a y cuya consistencia interna era de .87. En la muestra del presente trabajo la consistencia interna de los factores fue .91 y .86 respectivamente.

Además se utilizó una hoja de recogida de datos sociodemográficos y de salud así como, en el caso de las mujeres, de una serie de preguntas relativas a la menstruación que permitían conocer el periodo en que se encontraba en relación a su estatus menopáusico.

\section{Diseño}

Se ha utilizado un diseño transversal en el que las mujeres se han agrupado en función del estatus menopáusico que tenían al cumplimentar las pruebas y la edad en el caso de las mujeres más jóvenes. Partiendo de la definición de la OMS (1996) y de su edad, se formaron los siguientes grupos de mujeres:

- Grupo 1: Formado por 158 mujeres con edades comprendidas entre 20 y 31 años, sin amenorrea. Según las recomendaciones de la OMS en el uso de la terminología, estas mujeres se encuentran en periodo premenopáusico pero se diferencian de las del grupo 2 en que su edad es mucho menor y está claramente alejado de la edad a que tiene lugar la menopausia natural.

- Grupo 2: Formado por 352 mujeres con edades comprendidas entre 41 y 51 años que no presentan problemas menstruales ni amenorrea, por lo que se encuentran en periodo premenopáusico. Este grupo se denomina "Premenopausia/41 a 51 años".

- Grupo 3: Formado por 83 mujeres mayores de 40 años que se encuentran en fase de perimenopausia ya que, si bien no tienen amenorrea permanente, presentan variabilidad en el ciclo menstrual. A este grupo se le denomina "Perimenopausia/44 a 56 años” ya que sus edades están comprendidas entre los 44 y 56 años. 
- Grupo 4: Está formado por 133 mujeres que tienen menopausia natural, es decir, que ha pasado más de 12 meses desde su última menstruación y la ausencia de ésta no ha sido mediante procedimientos quirúrgicos ni químicos. Este grupo se denomina "Postmenopausial 52 a 65 años" ya que las mujeres que lo integran tienen edades comprendidas entre 52 y 65 años.

Los hombres se dividieron también en cuatro grupos de forma que sus edades fuesen equivalentes a las de los grupos de mujeres. Excepto en el grupo con edades comprendidas entre 20 y 31 años, que estaba formado por un total de 156 hombres, la asignación de los hombres a los grupos en que la edad se solapaba se hizo de forma aleatoria. El segundo grupo de hombres incluyó un total de 243 cuyas edades estaban entre 41 y 51 años, que era el rango de edad de las mujeres en premenopausia; un tercer grupo se formó con 84 hombres cuyo rango de edad era de 44 a 56, el mismo de las mujeres en fase de perimenopausia; y el tercero se formó por un total de 132 hombres cuyas edades se situaban entre 52 y 65 años, que correspondía al rango de edad del grupo de mujeres en fase de postmenopausia.

\section{Procedimiento y análisis estadístico}

Todas las personas que participaron en la presente investigación fueron voluntarias y no recibieron compensación económica por su participación. El acceso a la muestra fue a través de centros laborales, educativos y de asociaciones ciudadanas. El presente estudio se inserta dentro de una investigación más amplia sobre género y salud. Todas las pruebas fueron cumplimentadas de forma individual, generalmente autocumplimentadas si bien en las personas con bajo nivel de estudios, o en las que así lo preferían, se realizó en forma de entrevista estructurada llevada a cabo por estudiantes de psicología con formación en entrevista y en el pase de pruebas.
Los análisis estadísticos se realizaron con el paquete estadístico PASW Statistics 18. Para conocer si existían diferencias en el estado de salud mental y en autoestima en función del género en las distintas fases relativas al estatus menopáusico y/o de la edad se hicieron análisis multivariados de varianza multivariados (MANOVA) y univariados (ANOVA) en los que se consideraron como factores el género y las cuatro agrupaciones de estatus menopáusico-edad. También se hicieron MANOVAs y ANOVAs para analizar las diferencias dentro de cada género entre las distintas fases relativas al estatus menopáusico y/o de la edad, realizándose comparaciones post-hoc con ajuste de Bonferroni cuando se encontraron diferencias estadísticamente significativas.

\section{Resultados}

\section{Sintomatología mental}

En el MANOVA en que los factores fueron el género (hombre, mujer) y las cuatro agrupaciones de estatus menopáusico-edad (entre 20 y 31 años, premenopausia/edades entre 41 y 51 años, perimenopausia/edades entre 44 y 56 años, postmenopausia /edades entre 52 y 65 años) y las variables dependientes las puntuaciones en las cuatro escalas del GHQ-28, la interacción género X estatus menopáusicoedad fue estadísticamente significativa, $F(12,3519)=2.11, p$ $<.05$. Los ANOVA mostraron que las interacciones estadísticamente significativas se daban únicamente en las escalas de depresión grave, $F(3,1333)=3.49, p<.05$, y sintomatología somática, $F(3,1333)=4.11, p<.01$ (véase Figuras 1 y 2). A nivel multivariado también fueron estadísticamente significativos los efectos principales del género, $F(4,1330)$ $=15.29, p<.001 ; \mathrm{y}$ del estatus menopáusico-edad, $F(12$, 3519) $=3.20, p<.001$.

Tabla 1. Medias, desviaciones típicas y análisis de varianza en las escalas del GHQ-28 entre mujeres y hombres con diferente estatus menopáusico y/o edad.

\begin{tabular}{|c|c|c|c|c|c|c|c|}
\hline \multirow{2}{*}{ Grupo } & \multirow{2}{*}{ Escalas GHQ-28 } & \multicolumn{2}{|c|}{ MUJERES } & \multicolumn{2}{|c|}{ HOMBRES } & \multirow{2}{*}{$F$} & \multirow{2}{*}{$\begin{array}{c}\eta^{2} \\
\text { parcial }\end{array}$} \\
\hline & & $M$ & $D T$ & $M$ & $D T$ & & \\
\hline \multirow{4}{*}{ 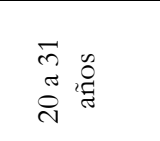 } & Síntomas somáticos & 6.37 & 3.84 & 5.22 & 3.70 & $7.30 * *$ & .023 \\
\hline & Ansiedad e insomnio & 7.16 & 4.86 & 6.03 & 4.61 & $4.54^{*}$ & .014 \\
\hline & Disfunción social & 10.39 & 3.25 & 10.62 & 2.91 & 0.89 & \\
\hline & Depresión grave & 2.68 & 3.65 & 2.56 & 3.68 & .07 & \\
\hline \multirow{4}{*}{ 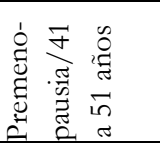 } & Síntomas somáticos & 6.20 & 4.17 & 5.05 & 3.78 & $11.6^{* *}$ & .019 \\
\hline & Ansiedad e insomnio & 6.73 & 4.82 & 5.17 & 3.70 & $118.2^{* * *}$ & .030 \\
\hline & Disfunción social & 11.22 & 2.88 & 11.12 & 2.64 & 0.19 & \\
\hline & Depresión grave & 2.39 & 3.75 & 1.40 & 2.36 & $13.3^{* * *}$ & .022 \\
\hline \multirow{4}{*}{ 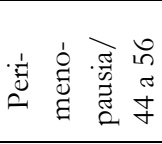 } & Síntomas somáticos & 7.86 & 4.80 & 4.36 & 3.58 & $28.6^{* * *}$ & .151 \\
\hline & Ansiedad e insomnio & 7.67 & 4.84 & 5.44 & 4.02 & $10.5^{* *}$ & .060 \\
\hline & Disfunción social & 11.17 & 2.97 & 11.20 & 2.54 & 0.01 & \\
\hline & Depresión grave & 3.01 & 3.58 & 0.90 & 1.51 & $24.6^{* * *}$ & .130 \\
\hline \multirow{4}{*}{ 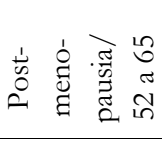 } & Síntomas somáticos & 6.63 & 4.34 & 5.40 & 3.99 & $5.76^{*}$ & .021 \\
\hline & Ansiedad e insomnio & 7.55 & 4.94 & 5.87 & 4.60 & $8.17 *$ & .030 \\
\hline & Disfunción social & 11.50 & 2.63 & 11.05 & 2.61 & 1.96 & \\
\hline & Depresión grave & 2.98 & 4.24 & 1.64 & 3.08 & $8.57 * * *$ & .032 \\
\hline
\end{tabular}


Para conocer la relevancia del género en los síntomas informados en cada grupo de edad y/o estatus menopáusico se hicieron análisis de varianza considerando como factor el género. El MANOVA realizado en el grupo de edad de entre 20 y 31 años evidenció la existencia de diferencias marginalmente significativas entre mujeres y hombres, $F(4,309)=$ 2.36, $p=.054$. Los ANOVA mostraron que solo se daban diferencias estadísticamente significativas en la sintomatología somática y de ansiedad e insomnio (véase Tabla 1).

Los MANOVA realizados en el resto de los grupos evidenciaron diferencias estadísticamente significativas en el grupo de premenopausia/edad entre 41 y 51, $F(4,590)=$ $5.32, p<.001$; en el de perimenopausia/edad entre 44 y 56 años, $F(4,162)=10.14, p<.001$; y en el de postmenopausia/edad entre 52 y 65 años, $F(4,260)=2.87, p<.05$. En la Tabla 1 se presentan las puntuaciones medias y los principales resultados de los análisis de varianza univariados realizados para cada grupo y en las Figuras 1 a 4 se muestra la representación gráfica de las puntuaciones medias, en cada grupo de edad/estatus menopáusico, de mujeres y hombres para cada escala del GHQ-28.

También se hicieron análisis de varianza para conocer si había diferencias dentro de cada género entre los diferentes grupos de edad/estatus menopáusico. El MANOVA realizado en el grupo de hombres en el que se consideró como factor los cuatro grupos de edad mostró diferencias estadísticamente significativas, $F(12,1608)=3.59, p<.001$. Los ANOVA para cada grupo de síntomas evidenciaron que las diferencias estadísticamente significativas solo se daban en sintomatología depresiva, $F(3,611)=7.98, p<.001$. Los análisis post hoc con ajuste de Bonferroni evidenciaron que tales diferencias se daban entre los de entre 20 y 31 años respecto a los tres grupos de mayor edad (véase Figura 1).

El MANOVA realizado para conocer si había diferencias en el grupo de mujeres en función del estatus menopáusico, y la edad en el caso de las más jóvenes, también mostró dife- rencias estadísticamente significativas, $F(12,1903)=2.28, p$ $<$.01. Los ANOVA evidenciaron que las diferencias estadísticamente significativas se limitaban a la sintomatología somática, $F(3,722)=3.58, p<.05$, y a disfunción social, $F(3$, $722)=4.11, p<.01$. Los análisis post-hoc con ajuste de Bonferroni evidenciaron diferencias estadísticamente significativas $(p<.05)$ en sintomatología somática entre las mujeres en premenopausia/edades entre 41 y 51 años con respecto a las que se encuentran en perimenopausia (véase Figura 2). Y las diferencias en disfunción social se daban entre las mujeres entre 20 y 31 años con respecto a las que se encontraban en premenopausia y sus edades estaban entre 41 y 51 años $(p<$ $.05)$, y a las que estaban en postmenopausia $(p<.01)$ (véase Figura 3).

La Figura 1 también permite observar las diferencias entre los géneros en sintomatología depresiva grave. Tal y como se muestra en la Tabla 1, excepto en las personas con edades entre 20 y 31 años, todas las diferencias entre mujeres y hombres son estadísticamente significativas.

La Figura 2 muestra las puntuaciones medias en sintomatología somática de mujeres y hombres en cada grupo, evidenciando la mayor sintomatología somática de las mujeres respecto a los hombres. Como puede observarse en la Tabla 1 , las diferencias son estadísticamente significativas en todos los grupos.

En la Figura 3 se representan las puntuaciones medias en disfunción social de mujeres y hombres en cada grupo. Como puede observarse en la Tabla 1, las diferencias entre mujeres y hombres no fueron estadísticamente significativas en ninguno de los grupos.

En la Figura 4 se representan las puntuaciones medias de las mujeres y hombres de cada grupo en la sintomatología de ansiedad e insomnio. Tal y como se muestra en la Tabla 1, las diferencias entre mujeres y hombres son estadísticamente significativas aunque, como se citó, no lo eran las diferencias intragénero.

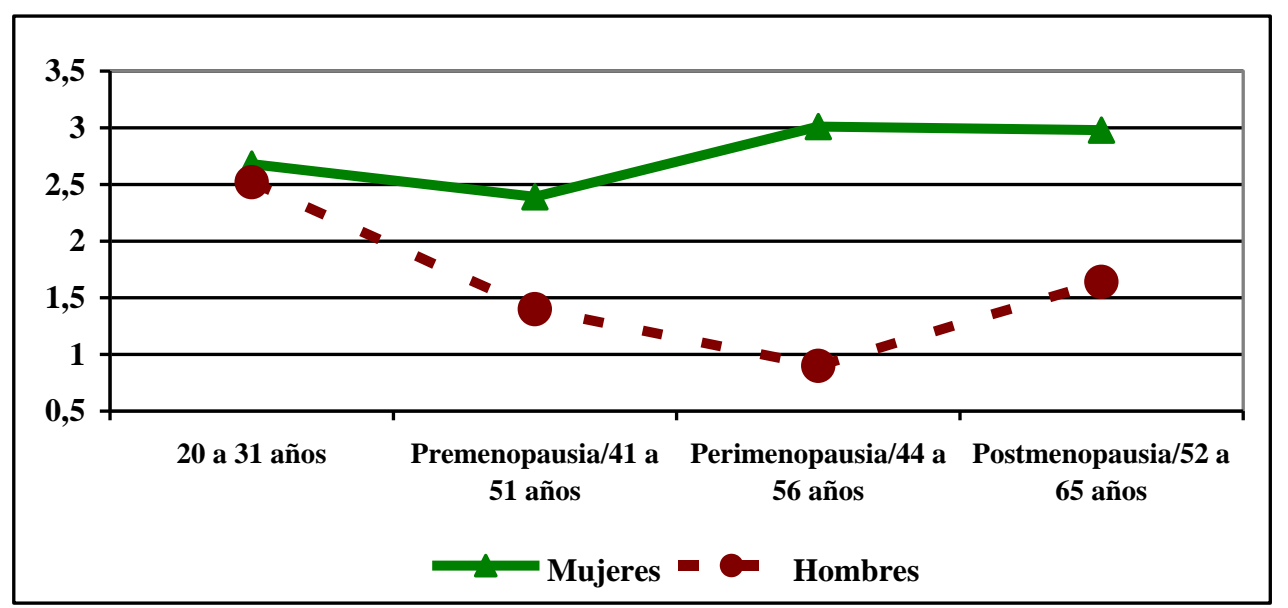

Figura 1. Síntomas de depresión grave en mujeres y hombres en función del estatus menopáusico y la edad. 


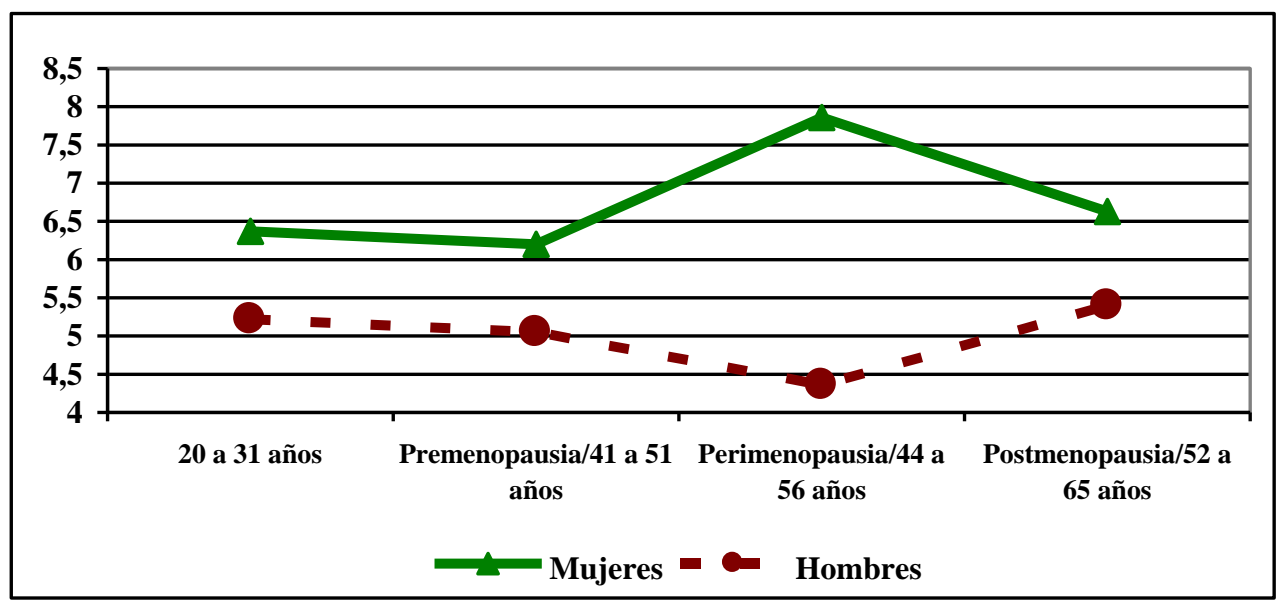

Figura 2. Sintomatología somática en mujeres y hombres en función del estatus menopáusico y la edad.

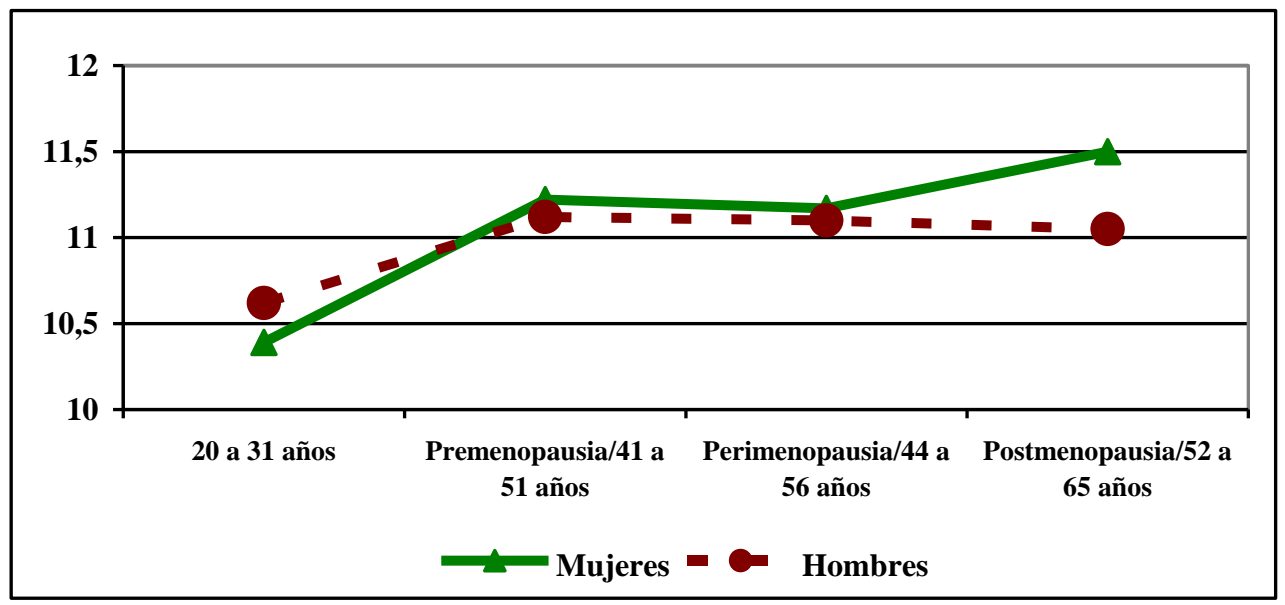

Figura 3. Disfunción social en mujeres y hombres en función del estatus menopáusico y la edad.

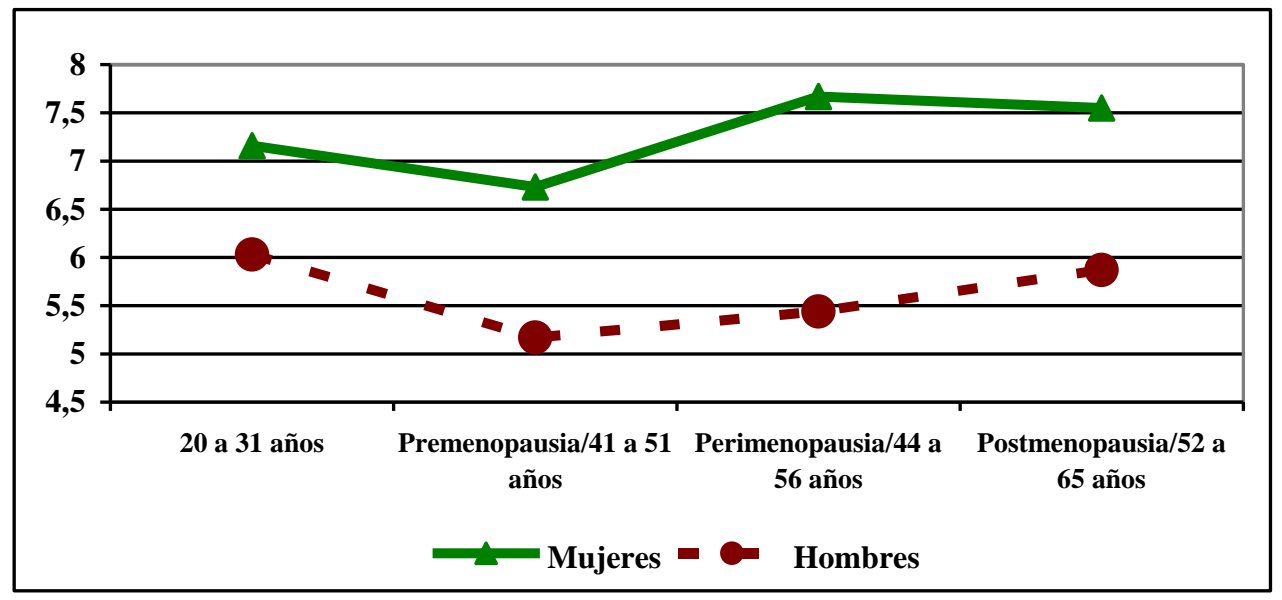

Figura 4. Síntomas de ansiedad e insomnio en mujeres y hombres en función del estatus menopáusico y la edad. 


\section{Diferencias en autoestima}

En el MANOVA en que las variables dependientes fueron los dos factores del cuestionario de autoestima, la interacción género $\mathrm{X}$ estatus menopáusico-edad fue estadísticamente significativa, $F(6,2644)=3.41, p<.01$. Los ANOVA mostraron que las interacciones estadísticamente signifi- cativas se daban en los dos factores: en valoración negativa de sí mismo/a, $F(3,1323)=2.86, p<.05$; y en autoconfianza, $F(3,1323)=5.76, p<.01$ (véase Figuras 5 y 6). En la Tabla 2 se muestran los principales resultados de los análisis de varianza realizados en cada grupo de edad/estatus menopáusico en que se consideró como factor el género.

Tabla 2. Medias, desviaciones típicas y análisis de varianza en los factores de autoestima (SEQ) entre mujeres y hombres con diferente estatus menopáusico y/o edad.

\begin{tabular}{|c|c|c|c|c|c|c|c|}
\hline \multirow[t]{2}{*}{ Grupos } & \multirow[t]{2}{*}{ Factores SEQ } & \multicolumn{2}{|c|}{ MUJERES } & \multicolumn{2}{|c|}{ HOMBRES } & \multirow{2}{*}{$F$} & \multirow{2}{*}{$\begin{array}{c}\eta^{2} \\
\text { parcial }\end{array}$} \\
\hline & & $M$ & $D T$ & $M$ & $D T$ & & \\
\hline \multirow[t]{2}{*}{20 a 31 años } & Valoración negativa de sí mismo/a & 25.15 & 12.88 & 25.27 & 12.57 & 0.01 & \\
\hline & Autoconfianza & 29.65 & 7.81 & 28.56 & 8.11 & 1.47 & \\
\hline \multirow{2}{*}{$\begin{array}{l}\text { Premenopausia/ } \\
41 \text { a } 51 \text { años }\end{array}$} & Valoración negativa de sí mismo/a & 24.36 & 12.56 & 22.01 & 10.76 & $5.62 *$ & .009 \\
\hline & Autoconfianza & 29.11 & 7.45 & 30.99 & 7.15 & $9.39 * *$ & .016 \\
\hline \multirow{2}{*}{$\begin{array}{l}\text { Perimenopausia/ } \\
44 \text { a } 56 \text { años }\end{array}$} & Valoración negativa de sí mismo/a & 25.85 & 13.31 & 19.17 & 9.62 & $13.5^{* * *}$ & .077 \\
\hline & Autoconfianza & 27.91 & 7.61 & 32.48 & 7.51 & $14.8^{* * *}$ & .084 \\
\hline \multirow{2}{*}{$\begin{array}{l}\text { Postmenopausia/ } \\
52 \text { a } 65 \text { años }\end{array}$} & Valoración negativa de sí mismo/a & 26.45 & 12.96 & 25.14 & 13.00 & 0.67 & \\
\hline & Autoconfianza & 29.08 & 7.68 & 31.57 & 7.51 & $7.15^{* * *}$ & .027 \\
\hline
\end{tabular}

Como puede observarse, los análisis de varianza realizados en el grupo de edad entre 20 y 31 años no mostraron la existencia de diferencias estadísticamente significativas ni a nivel multivariado, $F(2,313)=1,28, p>.05$, ni univariado. En el grupo de premenopausia/edad entre 41 y 51 años sí se encontraron diferencias estadísticamente significativas, tanto a nivel multivariado, $F(2,587)=4.97, p<.01$, como univariado. Lo mismo sucedió en el grupo de perimenopausia/edad entre 44 y 56 años, $F(2,160)=9.05, p<.001$. Y aunque se encontraron diferencias estadísticamente significativas en el MANOVA realizado en el grupo de postmenopausia/edad entre 52 y 65 años, $F(2,261)=3.84$, $p<.05$, solo fue estadísticamente significativo el ANOVA del factor de autoconfianza.

En las Figuras 5 y 6 se muestra la representación gráfica de las puntuaciones medias de mujeres y hombres en los dos factores del cuestionario de autoestima en cada grupo de edad/estatus menopáusico.

El MANOVA realizado para comparar los cuatro grupos de mujeres no mostró la existencia de diferencias estadísticamente significativas en función de la edad y/o el estatus menopáusico ni a nivel multivariado, $F(6,1432)=1.27, p>$ .05 , ni univariado, $F(3,716)=.97, p>.05$, en valoración negativa de sí misma; y $F(3,716)=.94, p>.05$, en autoconfianza. El MANOVA realizado en los hombres mostró dife- rencias estadísticamente significativas, $F(12,1212)=6.27, p$ $<$.001. Los ANOVA para cada factor mostraron diferencias estadísticamente significativas tanto en valoración negativa de sí mismo, $F(3,607)=7.01, p<.001$, como en autoconfianza, $F(3,607)=6.40, p<.001$.

Los análisis post hoc con ajuste de Bonferroni mostraron que se daban diferencias estadísticamente significativas $(p<$ .01) en el factor de valoración negativa de sí mismo entre los hombres con edades entre 44 y 56 años respecto a los de más edad y a los que tienen entre 20 y 31, grupo este último que también se diferenciaba significativamente $(p<.05)$ del de edades comprendidas entre 41 y 51 años.

Los análisis post hoc con ajuste de Bonferroni del factor de autoconfianza mostraron que las diferencias estadísticamente significativas $(p>.01)$ se daban entre los hombres con edades entre 20 y 31 y los otros tres grupos de mayor edad. Como se observa en la Figura 6, son los más jóvenes los que tienen menor confianza en sí mismos. $\mathrm{Y}$, aunque en las mujeres no se daban variaciones estadísticamente significativas en dicho factor entre las distintas etapas de su vida adulta, tenían significativamente menos confianza en sí mismas que los hombres con edades similares en todas las fases relativas a la menopausia, excepto cuando sus edades se situaban entre los 20 y 31 años (véase Tabla 2). 


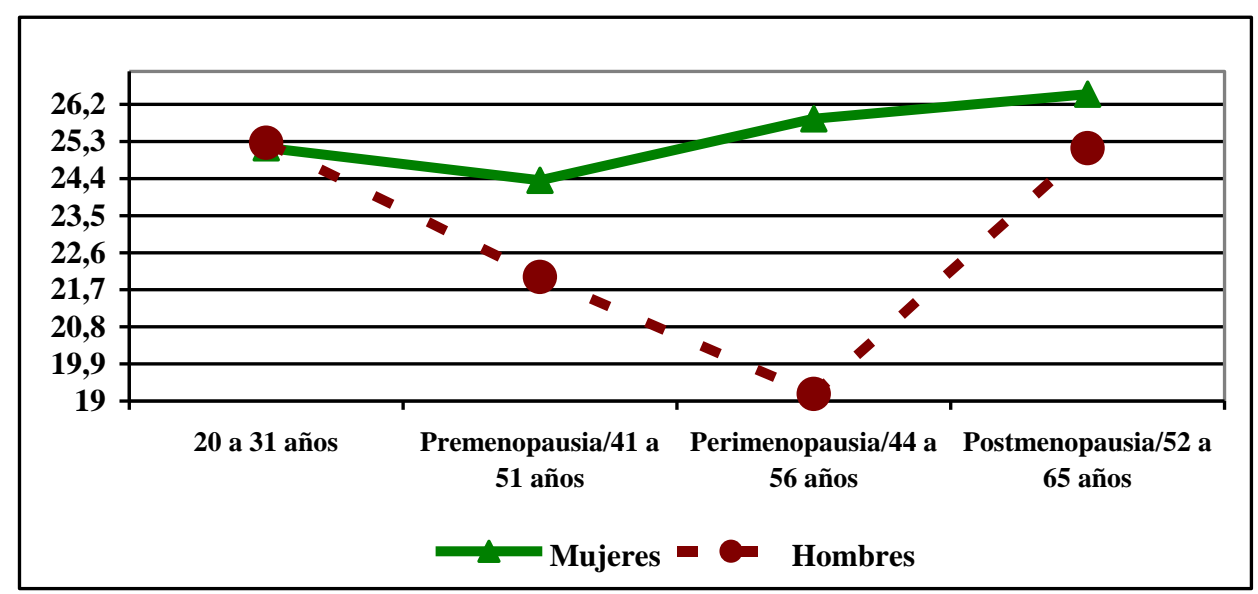

Figura 5. Valoración negativa de sí mismo/a en mujeres y hombres en función del estatus menopáusico y la edad.

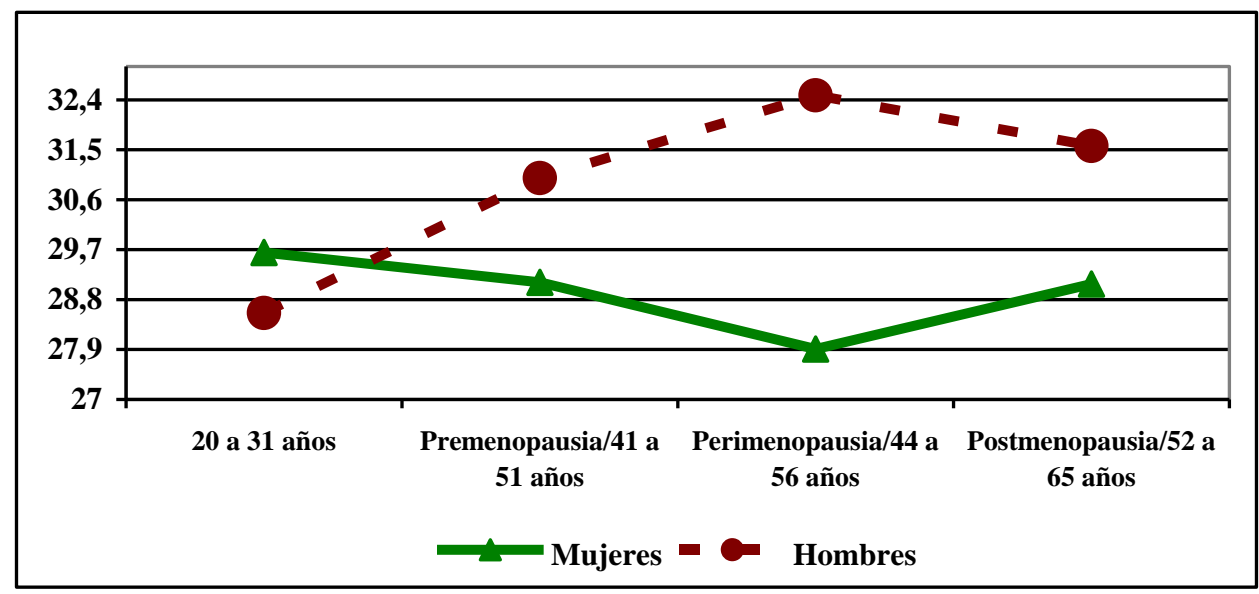

Figura 6. Autoconfianza en mujeres y hombres en función del estatus menopáusico y la edad.

\section{Discusión}

El objetivo del presente trabajo es analizar las diferencias entre mujeres y hombres a lo largo de la juventud y el periodo adulto en la sintomatología mental más común en la población y en autoestima, analizando la relevancia que el estatus menopáusico y la edad tienen en tales diferencias. Se ha encontrado que, aunque las mujeres tienen más sintomatología somática y de ansiedad e insomnio que los hombres a lo largo de todo el periodo de edad analizado (entre los 20 y 65 años), ello no sucede en sintomatología depresiva cuando las personas tienen edades entre 20 y 31 años. La ausencia de tales diferencias son coherentes con los resultados de un estudio reciente realizado en España (Jiménez-Torres, Martínez, Miró y Sánchez, 2012) donde se encontró que no había diferencias entre hombres y mujeres en el estado de ánimo negativo en una muestra de estudiantes con edades entre 17 y 25 años.

Se ha encontrado que, tanto en sintomatología somática como de depresión grave y en autoestima, el género interac- túa con la edad lo que indica que las trayectorias vitales de mujeres y hombres difieren en algunos indicadores de salud mental. Excepto en sintomatología de ansiedad e insomnio, donde no se han encontrado diferencias intragénero entre los 20 y 65 años ni en hombres ni en mujeres, se han detectado dentro de cada género diferencias a lo largo del ciclo vital estudiado en los indicadores de salud mental incluidos en el estudio, si bien las diferencias afectaban a variables distintas en mujeres y hombres. Así, en los análisis intragrupo realizados en la muestra de hombres, se han encontrado diferencias en función de la edad en sintomatología de depresión grave y en autoestima, siendo los más jóvenes (los de edades entre 20 y 31 años) los que presentan mayor sintomatología depresiva, mayor autovaloración negativa y menor confianza en sí mismos respecto a los que tienen más de 40 años. Por el contrario, en las mujeres, las diferencias intragénero se dan en sintomatología somática y en disfunción social pero no en sintomatología depresiva ni en autoestima. Y, mientras que en los hombres la dirección del cambio es hacia mejor salud mental en la adultez, en el caso de las mujeres parece suceder lo opuesto si bien tales diferencias parecen limitarse 
a disfunción social, que es menor en las mujeres más jóvenes. Además, las mujeres en perimenopausia informan de más sintomatología somática que el resto, si bien las diferencias solo son estadísticamente significativas respecto a las que están en el periodo anterior.

Frente a la consideración popular, y la perspectiva del modelo médico tradicional, de que la menopausia se asocia con sintomatología física y mental, los resultados del presente trabajo evidencian que solo parece darse un aumento en la sintomatología de tipo somático y únicamente durante el periodo de perimenopausia. Así se encontró que las mujeres en tal fase informaban de mayor cansancio, agotamiento, sensación de enfermedad, dolores de cabeza y de sensación de calor y/o escalofríos, pero no se detectó mayor sintomatología de ansiedad, de depresión ni menor autoestima. Son varios los estudios longitudinales realizados en otros países con muestras comunitarias que evidencian que la transición menopáusica no se asocia con depresión, (cfrs. Busch, Zonderman y Costa, 1994; Matthews et al., 1990) aunque en otros sí se ha encontrado mayor riesgo (Bromberger y Kravitz, 2011) y mayor sintomatología depresiva (Judd et al., 2012). En todo caso, estudios de revisión recientes plantean que, aunque se haya observado asociación entre menopausia y depresión, no existe una causa común (Llaneza et al., 2012) y los síntomas depresivos presentados no tiene significación diagnóstica específica (Judd et al., 2012). Y se reconoce que la mayoría de las mujeres pasan de los años de premenopausia a postmenopausia sin desarrollar sintomatología depresiva significativa (Soares, 2010).

Los resultados del presente estudio no apoyan las hipótesis biológicas que consideran que las diferencias entre mujeres y hombres en salud mental son debidas a las diferencias hormonales asociadas al sexo, excepto en lo relativo a la sintomatología de tipo somático. Los resultados encontrados más bien parecen apuntar, al menos en lo que a depresión y autoestima se refiere, a la relevancia de los roles sociales ya que no se dan diferencias entre mujeres y hombres al comienzo del periodo adulto pero sí surgen posteriormente. Y ello es debido a que los hombres presentan, en torno a los 40 y 50 años, una disminución importante de la sintomatología depresiva y un aumento de su autoestima, cambios que no parecen darse en las mujeres. La relevancia de los roles

\section{Referencias}

Ayers, B., Forshaw, M. y Hunter, M. (2010). The impact of attitudes towards the menopause on women's symptom experience: a systematic review. Maturitas 65, 28-36. doi:10.1016/j.maturitas.2009.10.016

Bebbington, P. E. (1998). Sex and depression. Psychological Medicine, 28, 1-8.

Bromberger, J. T. y Kravitz, H. M. (2011). Mood and Menopause: Findings from the Study of Women's Health Across the Nation (SWAN) over ten years. Obstetrics and Gynecology Clinics of North America, 38, 609-625. doi:10.1016/j.ogc.2011.05.011.

Busch, C. M., Zonderman, A. B. y Costa, P. T. (1994). Menopausal transition and psychological distress in a national representative simple: Is menopause associated with psychological distress? Journal of Aging and Health, 6, 209-228. sociales en las diferencias de género en depresión ha sido apuntada desde hace décadas (cfrs. Bebbington, 1998) habiéndose reconocido que tales diferencias aumentan en el periodo adulto cuando mujeres y hombres experimentan las desigualdad en estatus que conlleva el género (Mirowsky, 1996). El surgimiento de diferencias en depresión y autoestima entre mujeres y hombres en la adultez es una cuestión que debería analizarse en profundidad en posteriores trabajos, ya que dicho conocimiento puede ser útil para el diseño de políticas y programas que propicien mayor igualdad de género y mayor bienestar para la ciudadanía. Para el planteamiento de las variables sobre las que intervenir podría ser útil el análisis de los cambios en autoestima y sintomatología depresiva. Porque, aunque se conoce que la depresión se asocia con baja autoestima, aún no existe evidencia suficiente de si tal relación es porque la depresión disminuye la autoestima o porque la baja autoestima contribuye a la depresión, si bien recientemente se ha encontrado evidencia que apoya esta segunda perspectiva (Sowislo y Orth, 2013).

Este trabajo tiene una serie de limitaciones entre las que destaca el carácter transversal del estudio. Además, las personas participantes no se han obtenido mediante muestreo aleatorio, lo que limita la capacidad de generalización de los resultados. Pese a ello se evidencia que, al menos en España y para las mujeres que trabajan o participan en asociaciones ciudadanas, la menopausia no supone una amenaza importante para su salud mental. Porque, aunque se observa un aumento de síntomas de tipo somático durante la perimenopausia respecto a la premenopausia, dichos síntomas se limitan a ese periodo. Todo parece indicar que, tal y como plantean Busch et al. (1994), el estereotipo social de que la llegada de la menopausia se asocia con malestar, el cual perdura en muchas mujeres hasta varios años después, carece de apoyo empírico en las mujeres de la población general española. Por tanto, los resultados del presente estudio pueden ser útiles para ayudar a desmontar tales mitos así como para disminuir la percepción de la menopausia como un proceso altamente negativo percepción que, según Larroy y Gutiérrez (2009), se detecta en las mujeres españolas.

Agradecimientos.- Investigación subvencionada por el Ministerio de Ciencia e Innovación. Proyecto PSI2008-02543

Castaño, D. y Martínez-Benlloch, I. (1990). Aspectos psicosociales en el envejecimiento de las mujeres. Anales de Psicología, 6, 159-168.

Dawson, D. A., Goldstein, R. B., Moss, H. B., Li, T. y Grant, B. F. (2010). Gender differences in the relationship of internalizing and externalizing psychopathology to alcohol dependence: Likelihood, expression and course. Drug and Alcohol Dependence, 112, 9-17. doi:10.1016/j.drugalcdep.2010.04.019

Derry, P. S. (2002). What do we mean by "The biology of menopause". Sex Roles, 46, 13-23. doi:10.1023/A:1016081400820

Eaton, N. R., Keyes, K. M., Krueger, R. F., Balsis, S., Skodol, A. E., Markon. K. E., ... Hasin, D. S. (2012). An invariant dimensional liability model of gender gifferences in mental disorder prevalence: evidence 
from a national sample. Journal of Abnormal Psychology, 121, 282-288. doi: $10.1037 / \mathrm{a} 0024780$

Emslie, C., Fuhrer, R., Hunt, K., Macintyre, S., Shipley, M. y Stansfeld, S. (2002). Gender differences in mental health: evidence from three organizations. Social Science \& Medicine, 54, 621-624. doi:10.1016/S02779536(01)00056-9

Gallicchio L., Schilling, C., Miller, S. R., Zacur, H. y Flaws, J. A. (2007). Correlates of depressive symptoms among women undergoing the menopausal transition. Journal of Psychosomatic Research, 63, 263-268. doi:10.1016/j.jpsychores.2007.02.003

García-Vega, E. (2011). Acerca del género y la salud. Papeles del Psicólogo, 32, 282-288.

Gázquez, J. J., Yuste, N., y Pérez, M. C. (2005). Review of biological hypotheses explaining aging. Anales de Psicología, 21, 323-327.

Goldberg, D. P. y Hillier, V. F. (1979). A scaled version of the General Health Questionnaire. Psychological Medicine, 9, 139-145.

Goldberg, D., Williams, P., Lobo, A. y Muñoz, P. E. (1996). Cuestionario de Salud General (GHQ). Guia para el usuario de las distintas versiones. Barcelona: Masson.

Hall, L., Callister, L. C., Berry, J. A. y Matsumura, G. (2007). Meaning of menopause. Cultural influences on perception and management of menopause. Journal of Holistic Nursing, 25, 106-118. doi:10.1177/0898010107299432

Ishizuka, B., Kudo, Y. y Tango, T. (2008). Cross-sectional community survey of menopause symptoms among Japanese women. Maturitas, 61, 260-267. doi:10.1016/j.maturitas.2008.07.006

Jiménez-Torres, M. G., Martínez, M. P., Miró, E. y Sánchez, A. I. (2012). Relación entre estrés percibido y estado de ánimo negativo: diferencias según el estilo de afrontamiento. Anales de Psicología, 28, 28-36.

Judd, F. K., Hickey, M. y Bryant, C. (2012). Depression and midlife: Are we overpathologising the menopause? Journal of Affective Disorders, 136, 199211. doi:10.1016/j.jad.2010.12.010

Kessler, R. C., Chiu, W. T., Demler, O. y Walters, E. E. (2005). Prevalence, severity, and comorbidity of twelve-month DSM-IV disorders in the National Comorbidity Survey Replication (NCS-R). Archives of General Psychiatry, 62, 617-627. doi:10.1001/archpsyc.62.6.617

Kessler, R. C., McGonagle, K. A., Zhao, S., Nelson, C. B., Hughes, M. Eshleman, S., Wittchen, H. U. y Kendler, K. S. (1994). Lifetime and 12month prevalence of DSM-III-R psychiatric disorders in the United States: results from the National Comorbidity Survey. Archives of General Psychiatry, 51, 8-19.

Kroenke, K. y Spitzer, R. L. (1998). Gender differences in the reporting of physical and somatoform symptoms. Psychosomatic Medicine, 60. 150-155.

Lachowsky, M. (2002). Menopause: loss of self-esteem? International Congress Series, 1229, 25-30. doi:10.1016/S0531-5131(01)00472-1

Larroy, C. y Gutiérrez, S. (2009). Intervención cognitivo-conductual en sintomatología menopáusica: efectos a corto plazo. Psicothema, 21, 255-261.

Li, C., Samsioe, G., Borgfeldt, C., Lidfeldt, J., Agardh, C. D. y Nerbrand, C. (2003). Menopause-related symptoms: what are the background factors? A prospective population-based cohort study of Swedish women (The women's health in Lund area study). American Journal of Obstetrics and Gynecology, 189, 1643-1653. doi:10.1016/S0002-9378(03)00872-X

Llaneza, P., García-Portilla, M. P., Llaneza-Suárez, P. y Armott, B. (2012). Depressive disorder and the menopause transition. Maturitas, 71, 120130. doi:10.1016/j.maturitas.2011.11.017

Lund, K. L. (2008). Menopause and the menopausal transition. The Medical Clinics of North America, 92, 1253-1271. doi:10.1016/j.mcna.2008.04.009

Marcus-Newhall, A., Thompson, S. y Thomas, C. (2001). Examining a gender stereotype: menopausal women. Journal of Applied Social Psychology, 31, 698-719. doi:10.1111/j.1559-1816.2001.tb01409.x

Matthews, K. A., Wing, R. R., Kuller, L. H., Meilahn, E. N. , Kelsey, S. F., Costello y Caggiula, A. W. (1990). Influences of natural menopause on psychological characteristics and symptoms of middle-aged healthy women. Journal of Consulting and Clinical Psychology, 58, 345-351.

Matud, M. P., Ibáñez, I., Marrero, R. y Carballeira, M. (2003). Diferencias en autoestima en función del género. Análisis y Modificación de Conducta, 29, 51-78.

Mendoza, N., Sánchez-Borrego, R., Cancelo, M. J., Calvo, A., Checa, M. A., Cortés, J., Elorriaga, M. A., ... De la Viuda, E. (2013). Position of the Spanish menopause society regarding the management of perimenopause. Maturitas, 74, 283-290. doi:10.1016/j.maturitas.2012.12.010

Mirowsky, J. (1996). Age and the gender gap in depression. Journal of Health and Social Behavior, 37, 362-380.

Organización Mundial de la Salud (1981). Investigaciones sobre la Menopausia. Ginebra: OMS, Serie de informes técnicos: 670. Recuperado el 2 de febrero de 2013, de http://apps.who.int/iris/bitstream/10665/41316/1/WHO_TRS_670_ spa.pdf

Organización Mundial de la Salud (1996). Investigaciones sobre la Menopausia en los años noventa. Ginebra: OMS, Serie de informes técnicos: 866. Recuperado el 2 de febrero de 2013, de http://apps.who.int/iris/bitstream/10665/41984/1/WHO_TRS_866_ spa.pdf

Payne, J., Palmer, J. y Joffe, H. (2009). A reproductive subtype of depression: conceptualizing models and moving toward etiology. Harvard Review of Psychiatry 17, 72-86. doi:10.1080/10673220902899706

Pimenta, F., Leal, I., Maroco, J. y Ramos, C. (2012). Menopausal symptoms: do life events predict severity of symptoms in peri- and postmenopause? Maturitas, 72, 324-331. doi:10.1016/j.maturitas.2012.04.006

Rector, N. A, y Roger, D. (1993, julio). Self-concept and emotion-control. Presentación en el $3^{\text {rd }}$ Annual Meeting of the European Congress of Psychology. Helsinki, Finland.

Regier, D. A., Boyd, J. H., Burke, J. D., Rae, D. S., Myers, J. K., Kramer, M., ... Locke, B. Z. (1988). One-month prevalence of mental disorders in the United States. Archives of General Psychiatry, 45, 977-986. doi:10.1001/archpsyc.1988.01800350011002

Seedat, S., Scott, K. M., Angermeyer, M. C., Berlung, P., Bromet, E. J., Brugha, T. S., ... Kessler, R. C. (2009). Cross-National associations between gender and mental disorders in the World Health Organization World Mental Health Surveys. Archives of General Psychiatry, 66, 785-795. doi:10.1001/archgenpsychiatry.2009.36.

Sherman, S. (2005). Defining the menopausal transition. The American Journal of Medicine, 118, 3S-7S. doi:0.1016/j.amjmed.2005.11.002

Soares, C. N. (2010). Can depression be a menopause-associated risk? BioMed Central Medicine, 8, 79. Recuperado el 2 de noviembre de 2013, de http://biomedcentral.com/1741-7015/8/79.

Sowislo, J. F. y Orth, U. (2013). Does low self-esteem predict depression and anxiety? A meta-analysis of longitudinal studies. Psychological Bulletin, 139, 213-240. doi:10.1037/a0028931

Steiner, M., Dunn, E. y Born, L. (2003). Hormones and mood: from menarche to menopause and beyond. Journal of Affective Disorders, 74, 67-83. doi:10.1016/S0165-0327(02)00432-9

Ussher, J. M. (1992). Research and theory related to females reproduction: implications for clinical psychology. British Journal of Clinical Psychology, 31, 129-151. doi:10.1111/j.2044-8260.1992.tb00979.x

Utian, W. H. (2004). Menopause-related definitions. International Congress Series, 1266, 133-138. doi:10.1016/j.ucs.2004.01.102

Weich, S., Slogget, A. y Lewis, G. (1998). Social roles and gender difference in the prevalence of common mental disorders. British Journal of Psychiatry, 173, 489-493. doi:10.1192/bjp.173.6.489

(Artículo recibido: 21-5-2013; revisión recibida: 30-6-2013; aceptado: 4-8-2013) 